\title{
Evaluation of early aspect formation technique for aspect refactoring
}

\begin{abstract}
At the age of software modularization, Aspect-Oriented Software Development (AOSD) has become a central focus among researchers. In fact, recent developments in the aspectorientation has increased the need for refactoring on the existing applications. Although, source code level refactoring has high levels of preferences, it still remains ambiguous since the crosscutting concern is hard to be located at the extensive number of LOC. So, researchers have been seeking more attention on requirement level refactoring. However, these rapid changes are having a serious effect since there are continuous ambiguities on the concern handler at requirement level as well. The ambiguity caused by the incomplete aspect formation process at the requirement level itself. The incomplete formation of aspect at the requirement level, surely affect the flow of concern to the next software process phase. Therefore, a conceptual framework known as Requirement-Oriented Aspect Refactoring (ReqOAR) with the focus on aspect lexicon guideline (ALG) has been proposed. In particular, the proposed ALG has aided in mapping the textual elements with aspect idioms. Moreover, to prove the effectiveness of the proposed ALG, an experimental study was conducted. As a result, the study has shown the high significant value (P-value $<0.0005)$ on the accuracy in terms of precision of the aspect candidates which have been extracted using aspect lexicon guidelines as compared to the conventional technique.
\end{abstract}

Keyword: Aspect; Aspect lexicon; Aspect refactoring; Aspect-oriented requirement engineering; Early aspect 